\title{
Scalable Preparation of Multifunctional Fire-Retardant Ultralight Graphene Foams
}

Chuangang $\mathrm{Hu},{ }^{\dagger, \star,}$ Jiangli Xue, ${ }^{\dagger, \S}$ Liye Dong, ${ }^{\dagger}$ Yue Jiang, ${ }^{\dagger}$ Xiaopeng Wang, ${ }^{\dagger}$ Liangti Qu, ${ }^{* \dagger}$ Liming Dai*, 

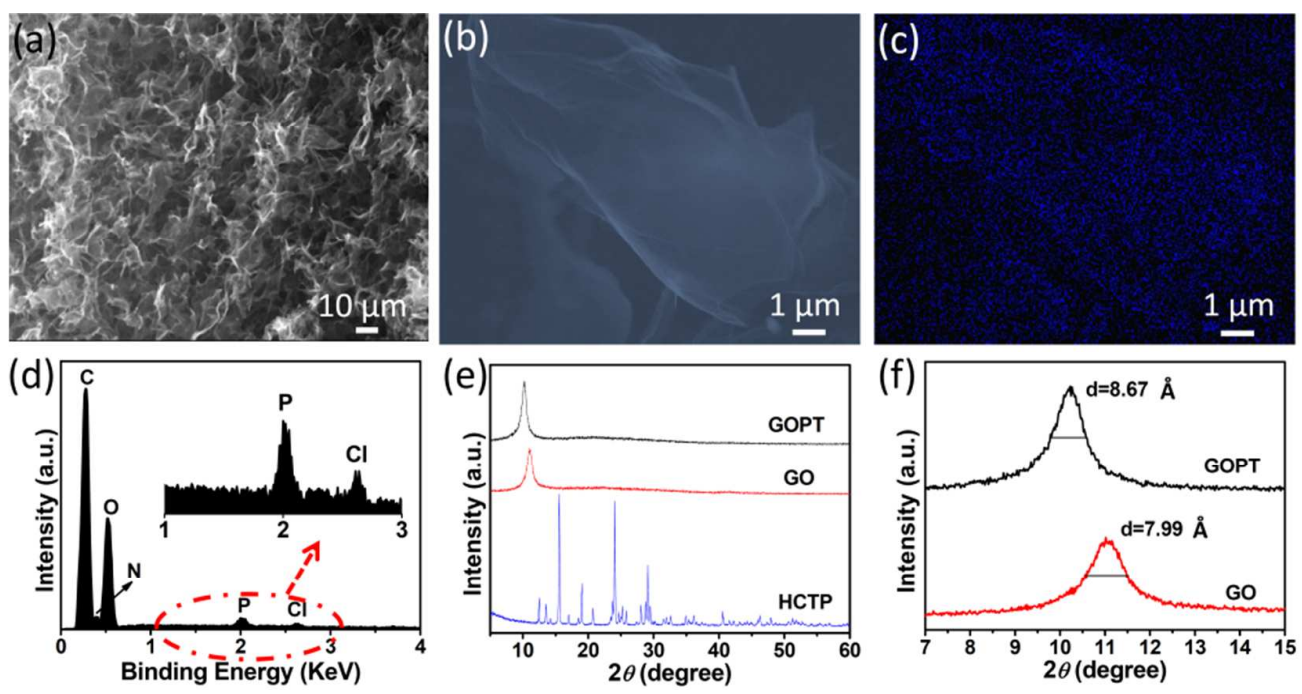

Figure S1. (a) SEM imaging of GOTP under low magnification. (b, c) SEM image and corresponding EDS mapping of P element. (d) Energy-dispersive spectroscopy (EDS) of the GOTP. (e) XRD pattern of GO and GOTP. (f) The enlargement of the (002) peaks of GO.

The GOTP foam exhibits a 3D porous structure (Figure S1a). The homogeneous distribution of $\mathrm{P}$ element on the GO sheets in the GOTP sample recorded by enlarged SEM imaging (Figure S1b) and the corresponding energy dispersive spectroscopy (EDS) mapping (FigureS1c), indicating that the HCTP organic compound is uniformly adsorbed on the sheets of 3D GO. The corresponding EDS (Figure S1d) reveals that the samples mainly composed of $\mathrm{C}, \mathrm{N}, \mathrm{P}$ and $\mathrm{Cl}$ elements as expected, plus $\mathrm{O}$ associated with GO. The inset in Figure S1d gives the amplified region of $\mathrm{P}$ and $\mathrm{Cl}$ for clear observation. As shown in Figure S1e, both X-ray diffraction (XRD) patterns of GO and GOTP exhibit a sharp diffraction peak at $2 \theta \approx 10^{\circ}$, and representative peaks of HCTP (the bottom curve in FigureS1e) has completely disappeared in the as-prepared GOTP samples. With the introduction of amount of HCTP, the diffraction peaks corresponding to the (002) plane of stacked sheets of GO and GOTP were gradually from $11.1^{\circ}$ shifted to smaller angle of $10.2^{\circ}$, indicating the 
enlargement of the interlayer distances. The interlayer spacing of GO and GOTP were calculated to be 7.99 and $8.67 \AA$, respectively (Figure S1f). It is reasonable to conclude that HCTP has been homogeneously intercalated into GO interlayers without phase separation.
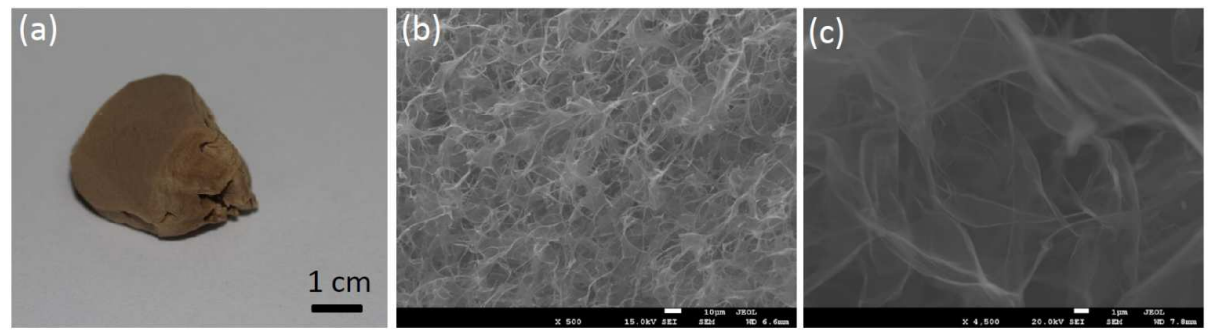

Figure S2. (a) Photograph GO foam. (b, c) SEM images of GO foam under different magnifications.

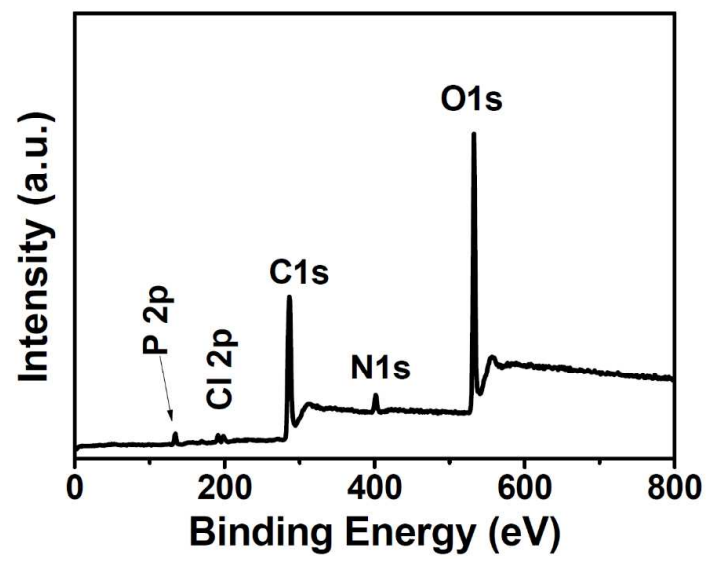

Figure S3. XPS survey of GOTP. The atomic ratio of C/O/P in the GOTP from XPS analysis is $c a$. $2.10 / 0.11 / 1 / 0.13$.

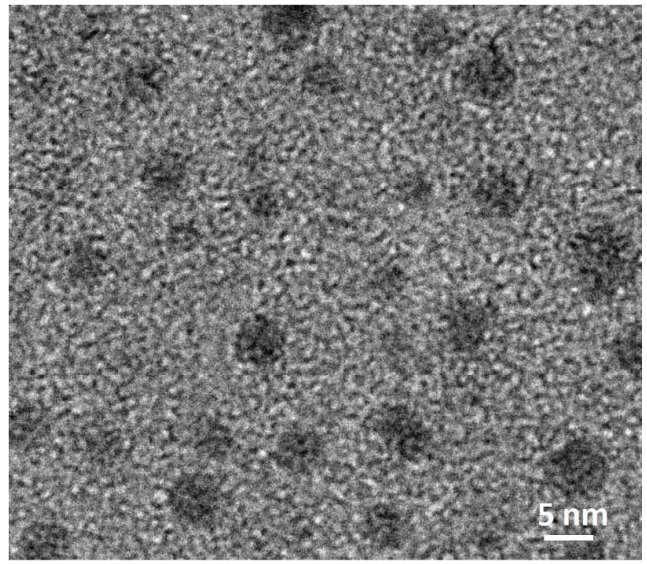

Figure S4.HR-TEM image of GPO. 


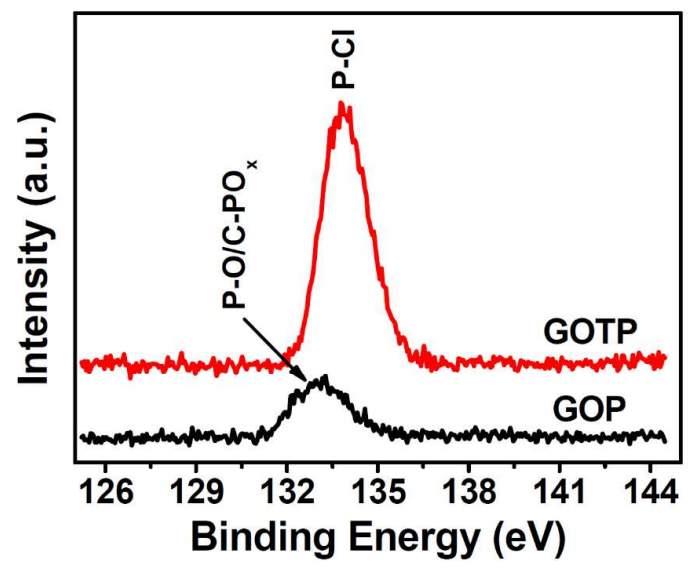

Figure S5. HR-XPS spectra of P elements in GOTP and GPO.

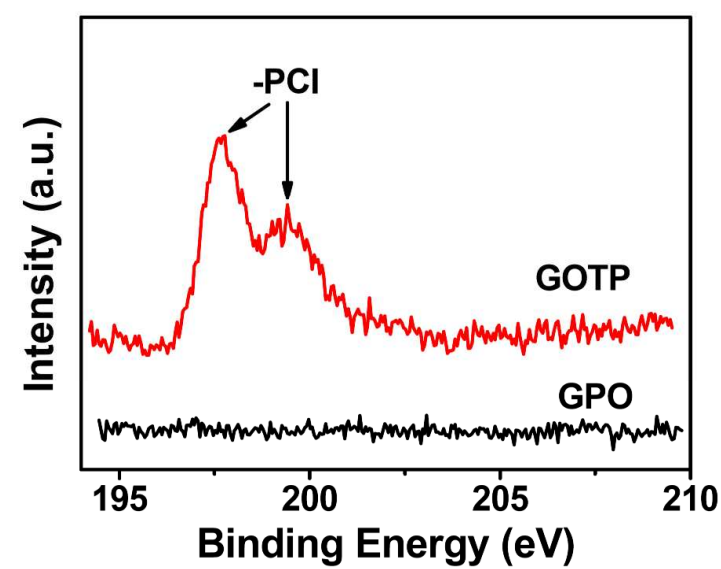

Figure S6. HR-XPS spectra of $\mathrm{Cl}$ elements in GOTP and GPO.

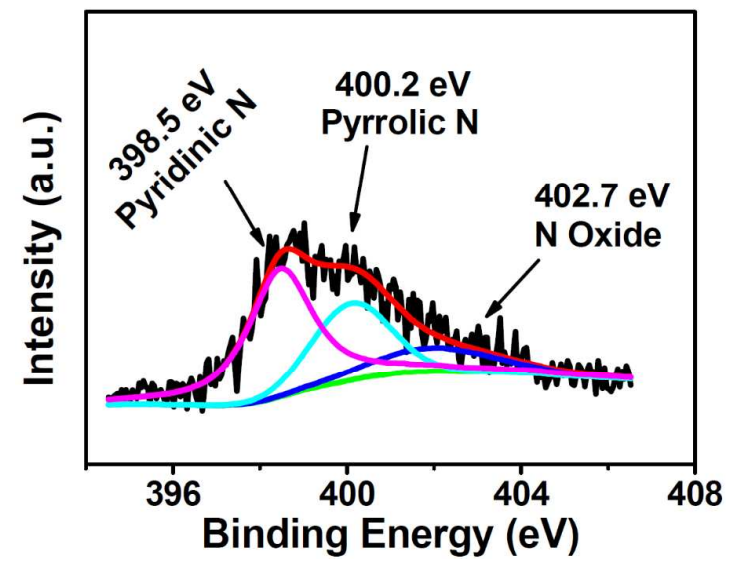

Figure S7. The high-resolution spectrum of N 1s XPS peak in GPO.

To confirm N-doping of graphene, X-ray photoemission spectroscopy (XPS) studies were carried out. The $\mathrm{N} 1 \mathrm{~s}$ peak can be resolved into three components centered at 398.5, 400.2 and $402.7 \mathrm{eV}$, representing pyridinic $\mathrm{N}$ atoms, pyrrolic $\mathrm{N}$ 
atoms, and $\mathrm{N}$ oxide ${ }^{[\mathrm{s} 1]}$ doped in the graphene structure (Figure S7).
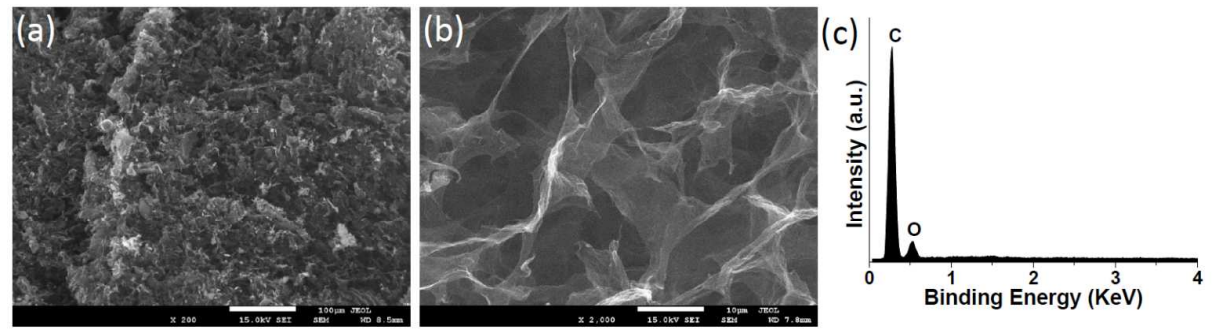

Figure S8. (a, b) SEM images of RGO after burning treatment. (c) The corresponding EDS spectrum.
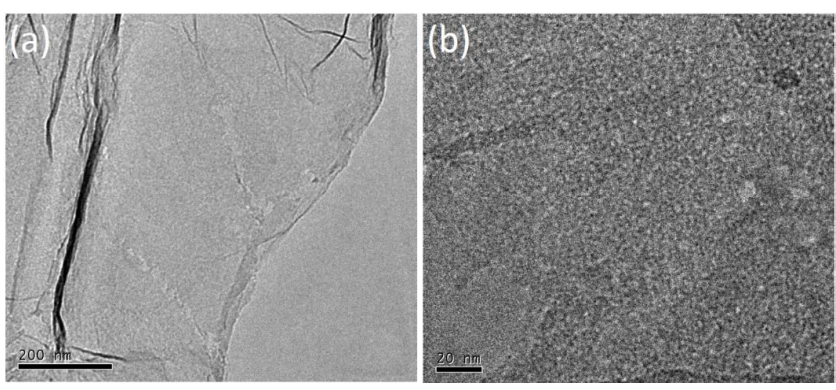

Figure S9. TEM imagings of RGO after burning treatment. There are some irregular holes on the surface of the graphene sheets, indicating the integrity of the structure may have been destroyed, which also could result in the instability.
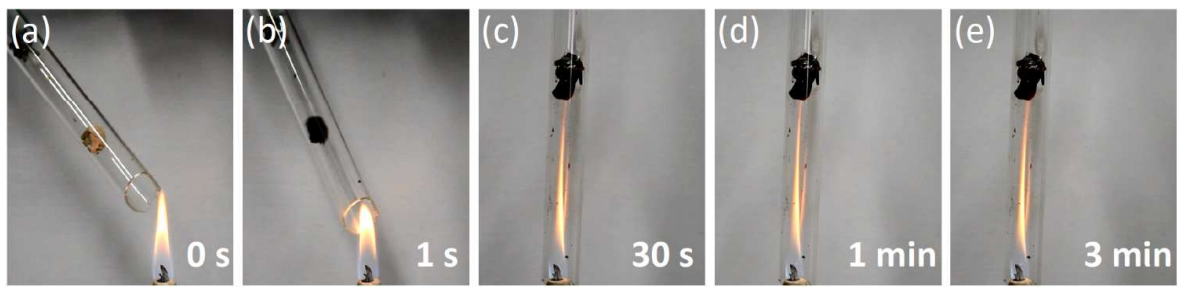

Figure S10. (a-e) Photographs of the GPO foam flow on the out flame with different times (the GPO was fixed in a quartz tube). From Figure S10a\&b, the GPOT was converted into GPO once it contacted with the flame. 

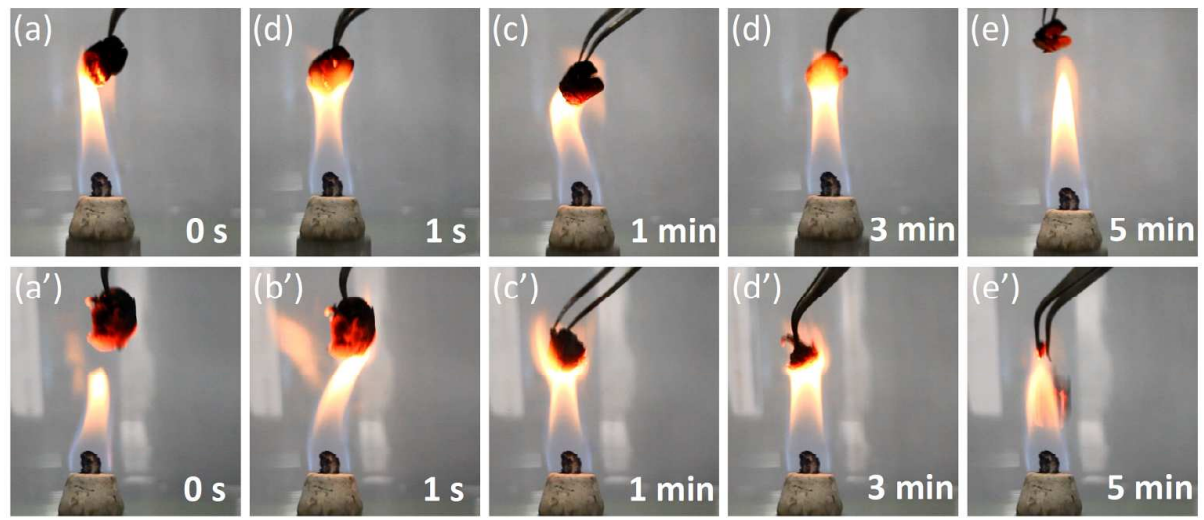

Figure S11. (a-e) Photographs of one GOTP foam on a hot flame with different times. (a'-e') Photographs of one GO foam on a hot flame with different times.
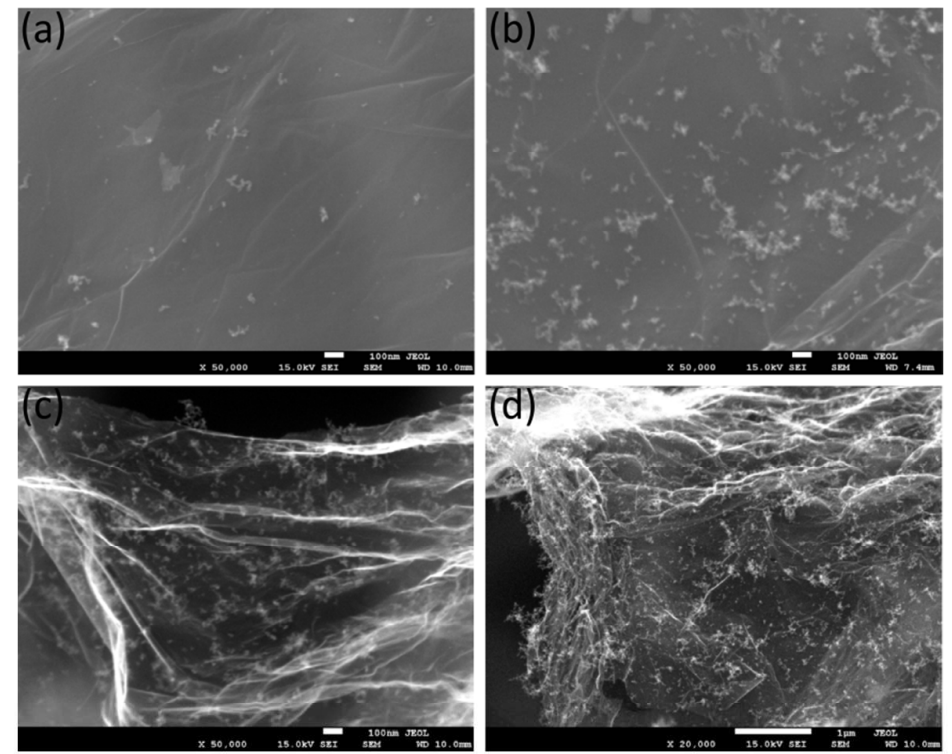

Figure S12. SEM images of GOTP samples with different mass ratios between GO and HCTP of (a) 20:1, (b) 10:1, (c) 5:1 and (d) 2.5:1, respectively, after heating on the flame with a temperature of $c a .500{ }^{\circ} \mathrm{C}$ for $20 \mathrm{~s}$. It is found that the density of $\mathrm{N}, \mathrm{P}$ contained particles are increased with the amount of HCPT. 


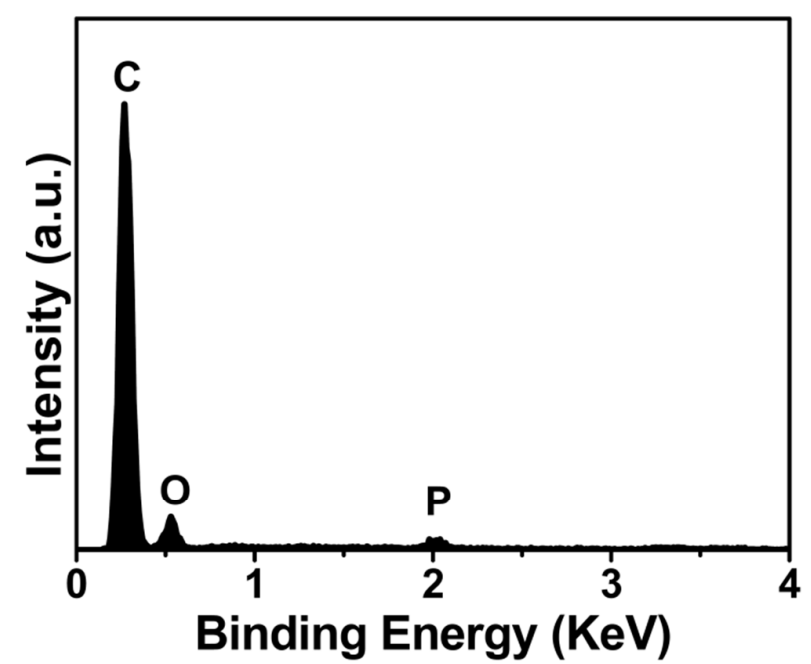

Figure S13. EDS of the GPO from the GOPT with the mass ratios between GO and HCTP of 2.5:1, and the content of $\mathrm{P}$ in the obtained GPO was $c a .1 .9 \%$.

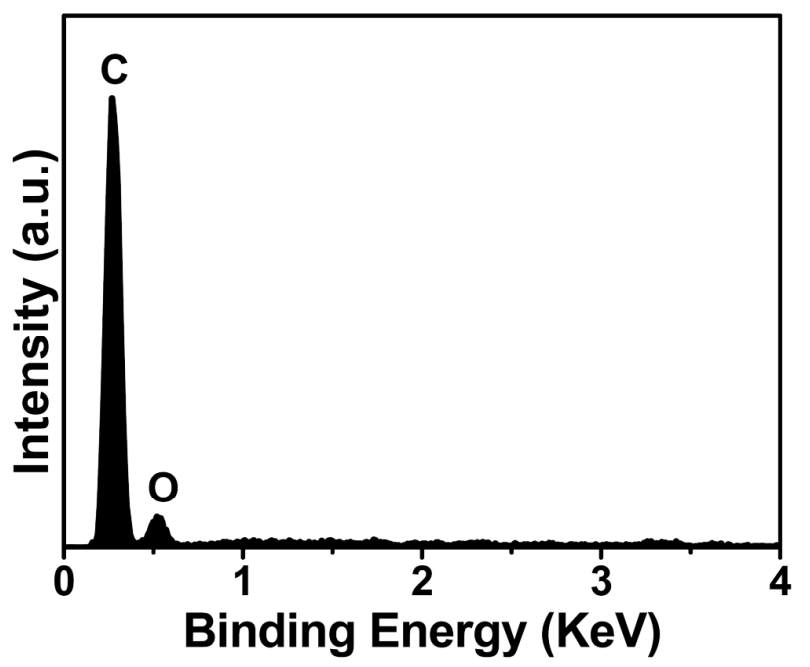

Figure S14. EDS of the GPO from the GOPT with the mass ratios between GO and HCTP of 20:1, and the content of $\mathrm{P}$ in the obtained GPO was almost negligible. 


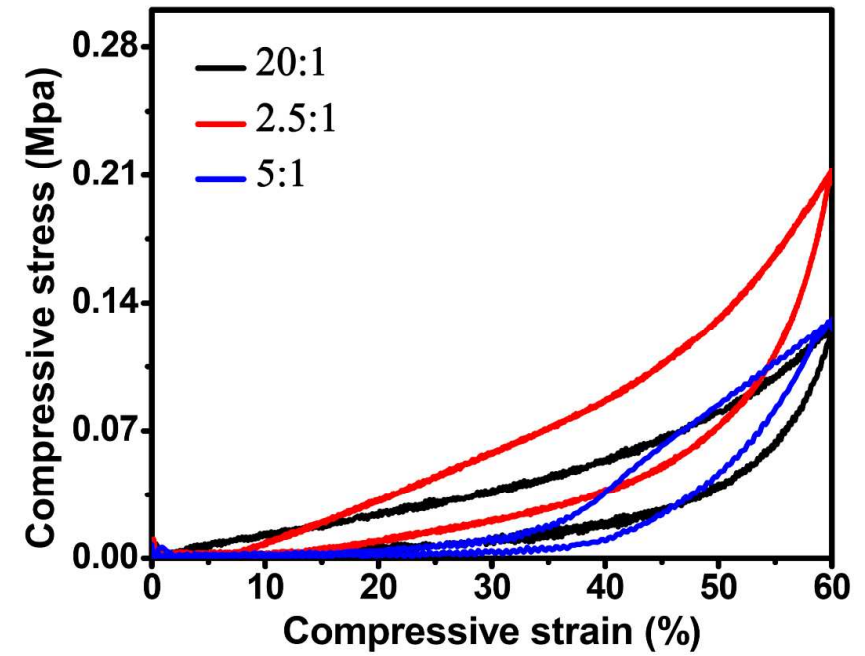

Figure S15. Stress-strain curves of GPO samples from the GOPT foams with different mass ratios between GO and HCTP of 20:1, 2.5:1 and 5:1, respectively. The set strain $(\varepsilon)$ is $60 \%$.

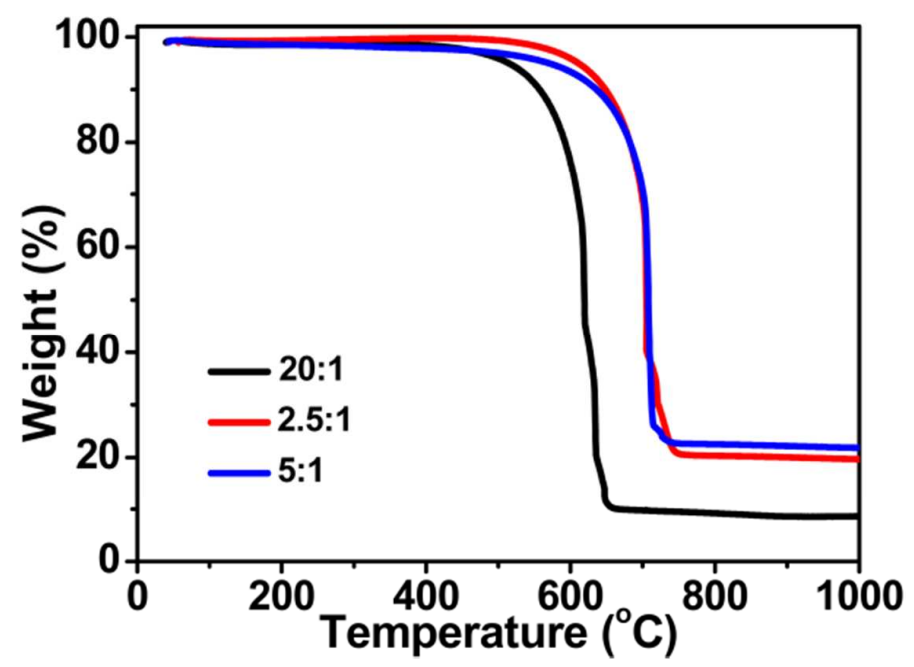

Figure S16. TGA curves of the GPO samples from the GOPT foams with different mass ratios between GO and HCTP of 20:1, 2.5:1 and 5:1, respectively. 

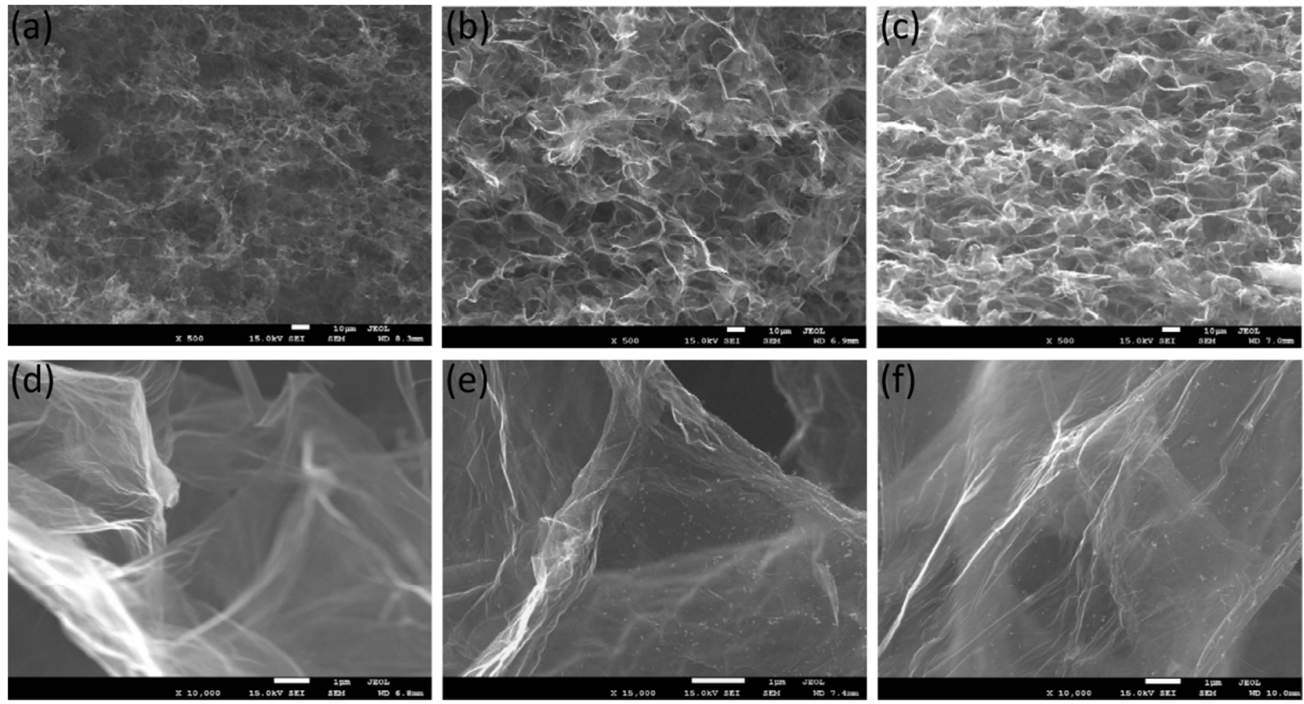

Figure S17. SEM images for the GPO samples from the GOPT foams with different mass ratios between GO and HCTP of (a, d) 20:1, (b, e) 2.5:1 and (c,f) 5:1, respectively.
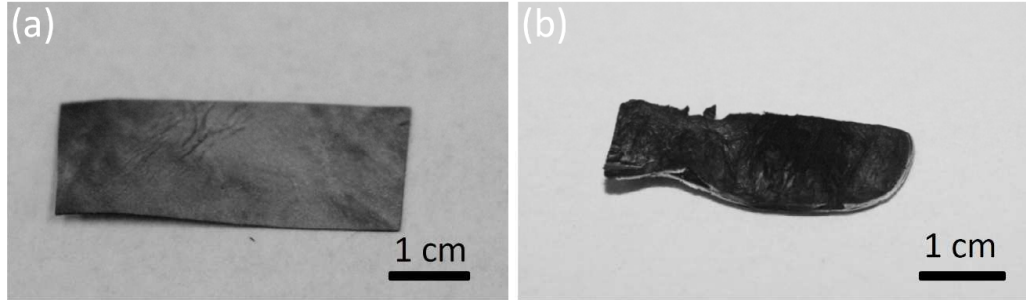

Figure S18. Photographs of GOTP film before and after burning. It can be seen that the shape of GPOT film maintained relative well even after burning on the outer flame of the alcohol lamp for $5 \mathrm{~min}$. 

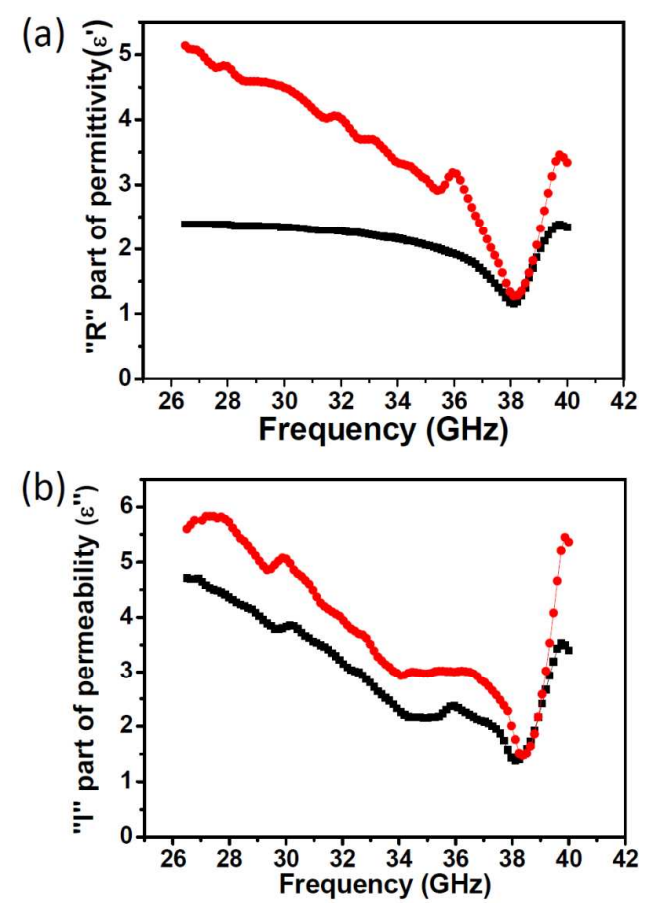

Figure S19. Frequency dependence of the (a) real and (b) imaginary parts of complex permittivity for GPO foam (red) before and (black) after burning $v s$. frequency.

Figure S19 shows the real and imaginary parts of the complex relative permittivity $\left(\varepsilon^{\prime}, \varepsilon^{\prime \prime}\right)$ of GPO before and afterburning on a hot flame. The value of the GPO after burning was slightly higher than that of the sample before burning, which means that RGO possesses enhanced dielectric loss after burning process. 

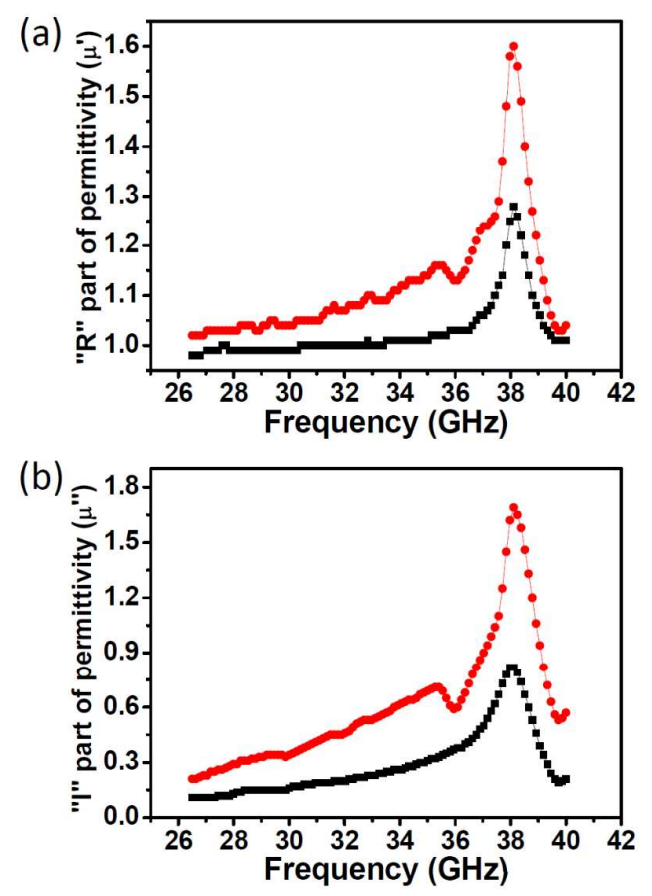

Figure S20. Frequency dependence of the (a) real and (b) imaginary parts of complex permeability for GPO foam (red) before and (black) after burning $v s$. frequency.

Figure S20 displays the real and imaginary parts of the complex permeability $\left(\mu^{\prime}\right.$, $\left.\mu^{\prime \prime}\right)$ measured for the two samples. The variation of both the real and imaginary parts of the complex permeability of GPO before and after burning on a hot flame, indicating the magnetic loss contribution to the microwave absorption of GPO after burning was slightly higher than before. 

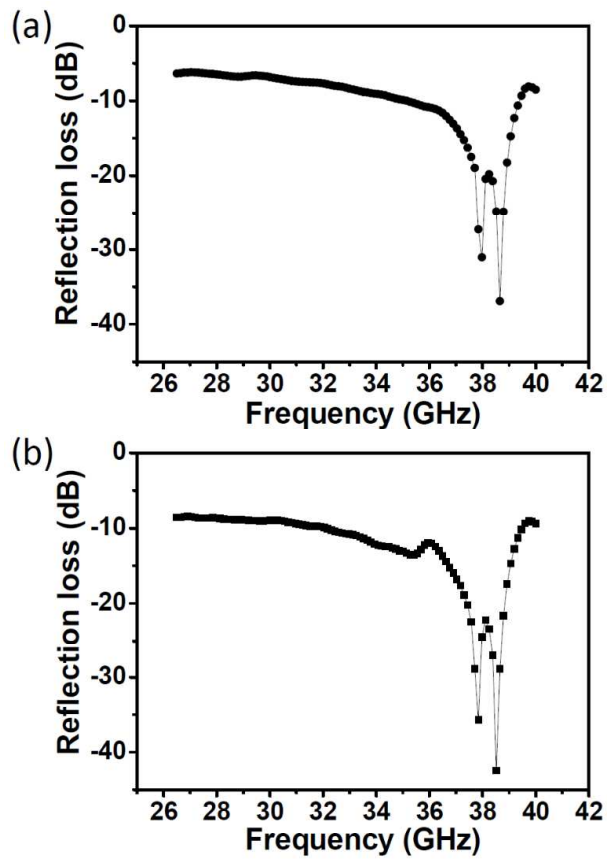

Figure S21. $(a, b)$ Comparison of the microwave absorption performance for GPO before and after burning on the flame with a thickness of $1.5 \mathrm{~mm}$.

Table S1. Comparison of flame retardant performance of different materials reported recently, including initial degradation temperature $\left(\mathrm{T}_{0}\right)$, the temperature of $10 \%$ decomposition and the maximum mass loss rate $\left(\mathrm{T}_{\max }\right)$, and char yield at $700{ }^{\circ} \mathrm{C}\left(\mathrm{Y}_{\mathrm{c}}\right.$ at $\left.700{ }^{\circ} \mathrm{C}\right)$.

\begin{tabular}{|c|c|c|c|c|c|}
\hline Samples & $\begin{array}{l}\mathrm{T}_{\mathbf{0}} \\
\left({ }^{\circ} \mathrm{C}\right)\end{array}$ & $\begin{array}{l}\mathrm{T}_{10} \\
\left({ }^{\circ} \mathrm{C}\right)\end{array}$ & $\begin{array}{l}\mathbf{T}_{\max } \\
\left({ }^{\circ} \mathrm{C}\right)\end{array}$ & $\begin{array}{l}\mathrm{Y}_{\mathrm{c}} \text { at } \\
700{ }^{\circ} \mathrm{C}\end{array}$ & Ref. \\
\hline GPO foam & 110 & 662 & 785 & $82 \%$ & This work \\
\hline $\begin{array}{l}\text { cellulose nanofibres-boric acid-sepiolite } \\
\text { nanorods-GO }\end{array}$ & 50 & 162 & $165 / 371$ & $53 \%$ & s2 \\
\hline $\begin{array}{c}\mathrm{N} \text {-aminoethyl piperazine and phosphonate } \\
\text { derivative functionalized GO }\end{array}$ & 213 & 308 & $338 / 607 / 723$ & $71 \%$ & s3 \\
\hline montmorillonite/G fibers & 479 & 628 & 682 & $31 \%$ & s4 \\
\hline
\end{tabular}




\begin{tabular}{|c|c|c|c|c|c|}
\hline polypropylene & 226 & 260 & 306 & 0 & s5 \\
\hline carbon fiber & 260 & 275 & 315 & $<8 \%$ & \\
\hline carbon black & 265 & 330 & 370 & $<8 \%$ & \\
\hline organic montmorillonite & 345 & 432 & 450 & 0 & s6 \\
\hline deoxyribonucleic acid(natural flame retardant) & 303 & 320 & $328 / 555$ & $5 \%$ & s7 \\
\hline rayon fibers & 50 & 326 & 323 & $18 \%$ & s8 \\
\hline ammonium polyphosphate (APP) & 295 & 375 & $420 / 610$ & $22 \%$ & s9 \\
\hline melamine (MEL) & 250 & 290 & 313 & $2 \%$ & \\
\hline pentaerythritol (PER) & 228 & 250 & 279 & $3 \%$ & \\
\hline acrylic resin & 137 & 261 & $215 / 362$ & $2 \%$ & \\
\hline $\begin{array}{l}\text { intumescent-flameretardant (IFR) } \\
\text { ethylene-vinyl acetate copolymer (EVA) }\end{array}$ & \multicolumn{4}{|c|}{ ethylene-vinyl acetate copolymer (EVA) } & $\mathrm{s} 10$ \\
\hline polybutyleneterephthalate & 340 & 390 & 400 & $5 \%$ & s11 \\
\hline epoxy resin (EP) & 400 & 400 & 420 & $15 \%$ & $\mathrm{~s} 12$ \\
\hline $\begin{array}{c}\mathrm{NO}_{3} \text {-functionalized layered double } \\
\text { hydroxides/epoxy resin }(\mathrm{LDH})\end{array}$ & 390 & 390 & 402 & $14 \%$ & \\
\hline \multicolumn{6}{|l|}{ sodium-LDH/EP } \\
\hline polypropylene (PP) & 330 & 398 & 430 & $27 \%$ & s13 \\
\hline ammonium polyphosphate (APP) & 280 & 332 & 440 & $5 \%$ & \\
\hline PP-wool-APP & 75 & 326 & 410 & $5 \%$ & \\
\hline polystyrene/magnesium & 105 & 112 & 337 & $30 \%$ & s14 \\
\hline \multicolumn{6}{|l|}{ hydroxide/encapsulated red phosphorus } \\
\hline dipentaerythritol & 202 & 321 & 360 & 0 & $\mathrm{~s} 15$ \\
\hline Melamine & 202 & 293 & 310 & 0 & \\
\hline ammonium polyphosphate & 280 & 420 & 470 & $<55 \%$ & \\
\hline
\end{tabular}




\begin{tabular}{|c|c|c|c|c|c|}
\hline 9,10-Dihydro-9-oxa-10-phosphaphenanthrene- & 70 & 450 & 470 & $40 \%$ & s 16 \\
\hline \multicolumn{6}{|l|}{ 10-oxide (DOPO)-polyhedral oligomeric } \\
\hline \multicolumn{6}{|l|}{ silsesquioxanes (POSS) } \\
\hline 4,40-bismaleimidophenyl methane & 240 & 469 & $480 / 620$ & $11 \%$ & \\
\hline \multicolumn{6}{|l|}{$(\mathrm{BDM}) / 2,20$-dially bisphenol A (DBA) } \\
\hline BDM/DBA/DOPO-POSS & 440 & 469 & $480 / 620$ & 0 & \\
\hline diglycidyl ether of bisphenol A & 312 & 378 & 590 & $52 \%$ & s17 \\
\hline Huntite/sodium alginate & 220 & 500 & $523 / 670$ & $51 \%$ & s 18 \\
\hline flame-retardant waterborne polyurethane & 150 & 265 & 340 & $17 \%$ & s19 \\
\hline starch & 120 & 320 & 330 & $15 \%$ & $\mathrm{~s} 20$ \\
\hline poly (butyl acrylate) & 35 & 382 & 396 & $12 \%$ & \\
\hline starch-g-poly(butyl acrylate) & 40 & 330 & 369 & $13 \%$ & \\
\hline ammonium polyphosphate/ethanolamine & 190 & 302 & $310 / 450$ & $<32 \%$ & $\mathrm{~s} 21$ \\
\hline bis(3-aminophenyl) methyl phosphine & 298 & 400 & $420 / 590$ & 0 & s22 \\
\hline \multicolumn{6}{|l|}{ oxide/bis(3-aminophenyl) phenyl phosphine } \\
\hline \multicolumn{6}{|l|}{ oxide (T20BD) } \\
\hline T20BD/DodecaPhenyl POSS & 298 & 400 & $420 / 590$ & $5 \%$ & \\
\hline T20BD/Epoxycyclohexyl POSS & 298 & 400 & $420 / 590$ & $4 \%$ & \\
\hline T20BD/Glycidyl POSS & 298 & 400 & $420 / 590$ & $4 \%$ & \\
\hline T20BD/Triglycidyl Cyclohexyl POSS & 298 & 400 & $420 / 590$ & $3 \%$ & \\
\hline Polyimide foams & 140 & 220 & $200 / 356$ & $55 \%$ & s 23 \\
\hline micro-sized hydrotalcites & 140 & 305 & 400 & $40 \%$ & \\
\hline expandable graphite (EG) & 141 & 303 & $485 / 717$ & $72 \%$ & $\mathrm{~s} 24$ \\
\hline microencapsulated EG & 141 & 303 & $484 / 714$ & $68 \%$ & \\
\hline polyurethane (PU) & 135 & 303 & $313 / 550$ & 0 & \\
\hline neat ammonium polyphosphate (APP) & 386 & & & & \\
\hline microencapsulated APP (MAPP) & 385 & & & & \\
\hline
\end{tabular}




\begin{tabular}{|c|c|c|c|c|c|}
\hline $\begin{array}{l}\text { polypropylene/intumescent flame } \\
\text { retardant/N-alkoxy hindered amine }\end{array}$ & 280 & 310 & 350 & $13 \%$ & s25 \\
\hline $\begin{array}{l}\text { poly(lactic acid)/hexa(phosphaphenanthrene } \\
\text { aminophenoxyl)-cyclotriphosphazene } \\
\text { composites }\end{array}$ & 45 & 53 & 120 & $<30 \%$ & s26 \\
\hline $\begin{array}{c}\text { polypropylene (PP)/ramie fiber (RF)-RF } \\
\text { composites }\end{array}$ & 100 & 124 & 200 & $<10 \%$ & s27 \\
\hline Bisphenol-A polycarbonate (PC) & 453 & 527 & $545 / 650$ & 0 & s28 \\
\hline $\begin{array}{l}\text { PC/sulfonate-containing polyhedral oligomeric } \\
\text { silsesquioxane }\end{array}$ & 394 & 488 & $490 / 596$ & 0 & \\
\hline High density polyethylene & 410 & 436 & 460 & 0 & s29 \\
\hline boric acid & 80 & 270 & 360 & $3 \%$ & \\
\hline borax & 130 & 350 & 360 & $11 \%$ & \\
\hline boric acid/borax & 130 & 360 & 360 & $2 \%$ & \\
\hline poly(sodium phosphate)/poly(allylamine) & 310 & 340 & 378 & 0 & s30 \\
\hline $\begin{array}{l}\text { poly(sodium phosphate)/poly(allylamine) } \\
\text { coated fabrics }\end{array}$ & 45 & 307 & 320 & $<25 \%$ & \\
\hline bannana pseudostem sap treated cotton & 63 & 175 & $320 / 445$ & $0.5 \%$ & $\mathrm{~s} 31$ \\
\hline $\begin{array}{l}\text { nitrogen-modified silane hybrid ( } \mathrm{SiN}) \text { and } \\
\text { phytic acid (PA) deposited cotton }\end{array}$ & 100 & 300 & 310 & $37 \%$ & $\mathrm{~s} 32$ \\
\hline Zinc N-morpholinomethylphosphonicacid & 397 & 470 & 487 & $66 \%$ & s33 \\
\hline EVA -aluminum trihydroxide (ATH) & 150 & 275 & $264 / 306 / 44$ & $32 \%$ & s34 \\
\hline EVA-ATH-melamine (MEL) & 150 & 263 & $270 / 310 / 452$ & $28 \%$ & \\
\hline aluminum trihydroxide & 218 & 283 & 289 & $<65 \%$ & $\mathrm{~s} 35$ \\
\hline $\begin{array}{l}\text { poly(ethylene-co-acrylic acid)/magnesium } \\
\text { di-hydroxide }\left(\mathrm{Mg}(\mathrm{OH})_{2}\right)\end{array}$ & 38 & 84 & $74 / 275 / 345$ & $56 \%$ & s36 \\
\hline poly(lactic acid)/Na-phytates & 275 & 350 & 380 & $<16 \%$ & s37 \\
\hline
\end{tabular}


Note: GO and G stand for graphene oxide and reduced graphene oxide (also graphene), respectively.

\section{References}

s1. Yang, S. Y.; Chang, K. H.; Huang, Y. L.; Lee, Y. F.; Tien, H. W.; Li, S. M.; Lee, Y. H.; Liu, C. H.; Ma, C. C.; Hu, C. C. A Powerful Approach to Fabricate Nitrogen-Doped Graphene Sheets with High Specific Surface Area. Electrochem. Commun. 2012, 14, 39-42.

s2. Wicklein, B.; Kocjan, A.; Salazar-Alvarez, G.; Carosio, F.; Camino, G.; Antonietti, M.; Bergström, L. Observation of Viscoelasticity in Boron Nitride Nanosheet Aerogel. Nat. Nanotech. 2015, 10, 277-283.

s3. Hu, W. Z.; Yu, B.; Jiang, S. D.; Song, L.; Hu, Y.; Wang, B. B. Hyper-Branched Polymer Grafting Graphene Oxide as an Effective Flame Retardant and Smoke Suppressant for Polystyrene. J. Hazard. Mater. 2015, 300, 58-66.

s4. Fang, B.; Peng, L.; Xu, Z.; Gao, C. Wet-Spinning of Continuous Montmorilonite-Graphene Fibers for Fire-Resistant Lightweight Conductors. ACS Nano 2015, 9, 5214-5222.

s5. Yang, H. F.; Gong, J.; Wen, X.; Xue, J.; Chen, Q.; Jiang, Z. W.; Tian, N. N.; Tang, T. Effect of Carbon Black on Improving Thermal Stability, Flame Retardancy and Electrical Conductivity of Polypropylene/Carbon Fiber Composites. Compos. Sci. Technol. 2015, 113, 31-37.

s6. Guo, J. B.; Xu, Y.; Chen, X. L.; Hu, S. J.; He, M.; Qin, S. H. Influences of Organic 
Montmorillonite on the Combustion Behaviors and Thermal Stability of Polyamide 6/Polystyrene Blend. High Perform. Polym. 2015, 27, 392-401.

s7. Alongi, J.; Blasio, A. D.; Milnes, J.; Malucelli, G.; Bourbigot, S.; Kandola, B.; Camino, G. Thermal Degradation of DNA, an All-in-One Natural Intumescent Flame Retardant. Polym. Degrad. Stab.2015, 113, 110-118.

s8. Frank, E.; Steudle, L. M.; Ingildeev, D.; Spörl, J. M.; Buchmeiser, M. R. Carbon Fibers: Precursor Systems, Processing, Structure, and Properties. Angew. Chem. Int. Ed. 2014, 53, 5262-5298.

s9. Beheshti, A.; Heris, S. Z. Experimental Investigation and Characterization of an Efficient Nanopowder-Based Flame Retardant Coating for Atmospheric-Metallic Substrates. Powder Technol. 2015, 269, 22-29.

s10. Zhang, F.; Sun, W. Y.; Wang, Y.; Liu, B. S. Influence of the Pentaerythritol Phosphate Melamine Salt Content on the Combustion and Thermal Decomposition Process of Intumescent Flame-Retardant Ethylene-Vinyl Acetate Copolymer Composites. J. Appl. Polym. Sci. 2015, 132, 42148.

s11. Hooshangi, Z.; Feghhi, S. A. H.; Sheikh, N. The Effect of Electron-Beam Irradiation and Halogen-Free Flame Retardants on Properties of Poly Butylene Terephthalate. Radiat. Phys. Chem.2015, 108, 54-59.

s12. Kalali, E. N.; Wang, X.; Wang, D. Y. Functionalized Layered Double Hydroxide-Based Epoxy Nanocomposites with Improved Flame Retardancy and Mechanical Propertie. J. Mater. Chem. A 2015, 3, 6819-6826.

S3. Kim, N. K.; Lin, R. J. T.; Bhattacharyya, D. Effects of Wool Fibres, Ammonium 
Polyphosphate and Polymer Viscosity on the Flammability and Mechanical Performance of PP/Wool Composites. Polym. Degrad. Stab. 2015, 119, 167-177.

s4. Yu, Z. L.; Liu, J. C.; Zhang, Y. B.; Luo, J.; Lu, C.; Pan, B. L. Thermo-Oxidative Degradation Behavior and Fire Performance of High Impact Polystyrene/Magnesium Hydroxide/Microencapsulated Red Phosphorus Composite with an Alternating Layered Structure. Polym. Degrad. Stab. 2015, $115,54-62$.

s15. Kahraman, H. T.; Gevgilili, H.; Pehlivan, E.; Kalyon, D. M. Development of an Epoxy Based Intumescent System Comprising of Nanoclays Blended with Appropriate Formulating Agents. Prog. Org. Coat. 2015, 78, 208-219.

s16. Wang, Z.; Wu, W.; Zhong, Y. H.; Ruan, M. Z.; Hui, L. L. Flame-Retardant Materials Based on Phosphorus-Containing Polyhedral Oligomeric Silsesquioxane and Bismaleimide/Diallylbisphenol a with Improved Thermal Resistance and Dielectric Properties. J. Appl. Polym. Sci. 2015, 132, 41545.

s17. Laachachi, A.; Burger, N.; Apaydin, K.; Sonnier, R.; Ferriol, M. Is Expanded Graphite Acting as Flame Retardant in Epoxy Resin? Polym. Degrad. Stab. 2015, $117,22-29$.

s18. Zhang, T. S.; Luo, D.; Wojtal, P.; Zhitomirsky, I. Electrophoretic Deposition of Flame Retardant Polymer-Huntite Coatings. Mater. Lett. 2015, 159, 106-109.

s19. Gu, L. M.; luo, Y. J. Flame Retardancy and Thermal Decomposition of Phosphorus-Containing Waterborne Polyurethanes Modified by Halogen-Free Flame Retardants. Ind. Eng. Chem. Res. 2015, 54, 2431-2438. 
s20. Mallick, B. N.; Rana, P. K.; Sahoo, P. K. Preparation of Starch-Based Bionanocomposite Hydrogel with Mica for Fire Retardancy. Adv. Polym. Technol. $\mathbf{2 0 1 5}, 34,21520$.

s21. Guan, Y. H.; Huang, J. Q.; Yang, J. C.; Shao, Z. B.; Wang, Y. Z. An Effective Way to Flame-Retard Biocomposite with Ethanolamine Modified Ammonium Polyphosphate and Its Flame Retardant Mechanisms. Ind. Eng. Chem. Res. 2015, $54,3524-3531$.

s22. Raimondo, M.; Russo, S.; Guadagno, L.; Longo, P.; Chirico, S.; Mariconda, A.; Bonnaud, L.; Murariu, O.; Dubois, P. Effect of Incorporation of POSS Compounds and Phosphorous Hardeners on Thermal and Fire Resistance of Nanofilled Aeronautic Resins. RSC Adv. 2015, 5, 10974-10986.

s23. Sun, G. H.; Liu, L. H.; Wang, J.; Wang, H. L.; Wang, W. P.; Han, S. H. Effects of Hydrotalcites and Tris (1-chloro-2-propyl) Phosphate on Thermal Stability, Cellular Structure and Fire Resistance of Isocyanate-Based Polyimide Foams. Polym. Degrad. Stab. 2015, 115, 1-15.

s24. Gao, L. P.; Zheng, G. Y.; Zhou, Y. H.; Hu, L. H.; Feng, G. D. Thermal Performances and Fire Behaviors of Rosin-Based Rigid Polyurethane Foam Nanocomposites. J. Therm. Anal. Calorim. 2015, 119, 411-424.

s25. Xie, H. L.; Lai, X. J.; Zhou, R. M.; Li, H. Q.; Zhang, Y. J.; Zeng, X. R.; Guo, J. H. Effect and Mechanism of N-Alkoxy Hindered Amine on the Flame Retardancy, UV Aging Resistance and Thermal Degradation of Intumescent Flame Retardant Polypropylene. Polym. Degrad. Stab. 2015, 118, 167-177. 
s26. Jiang, P.; Gu, X. Y.; Zhang, S.; Wu, S. D.; Zhao, Q.; Hu, Z. W. Synthesis, Characterization, and Utilization of a Novel Phosphorus/Nitrogen-Containing Flame Retardant. Ind. Eng. Chem. Res. 2015, 54, 2974-2982.

s27. Du, S. L.; Lin, X. B.; Jian, R. K.; Deng, C.; Wang, Y. Z. Chin. Flame-Retardant Wrapped Ramie Fibers towards Suppressing "Candlewick Effect" of Polypropylene/Ramie Fiber Composites. J. Polym. Sci. 2015, 33, 84-94.

s28. Li, Z. Q.; Yang, R. J. Flame Retardancy, Thermal and Mechanical Properties of Sulfonate-Containing Polyhedral Oligomeric Silsesquioxane (S-POSS)/Polycarbonate Composites. Polym. Degrad. Stab. 2015, 116, 81-87.

s29. Cavdar, A. D.; Mengeloğlu, F.; Karakus, K. Effect of Boric Acid and Borax on Mechanical, Fire and Thermal Properties of Wood Flour Filled High Density Polyethylene Composites. Meas. 2015, 60, 6-12.

s30. Li, Y. C.; Mannen, S.; Morgan, A. B.; Chang, S. C.; Yang, Y. H.; Condon, B.; Grunlan, J. C. Intumescent All-Polymer Multilayer Nanocoating Capable of Extinguishing Flame on Fabric. Adv. Mater. 2011, 23, 3926-3931.

s31. Basak, S.; Samanta, K. K.; Chattopadhyay, S. K.; Narkar, R. S.; Mahangade, R. Flame Retardant Cellulosic Textile using Bannana Pseudostem Sap. Int. J. Cloth. Sci. Tech. 2015, 27, 247-261.

s32. Wang, X.; Romero, M. Q.; Zhang, X. Q.; Wang, R.; Wang, D. Y. Intumescent Multilayer Hybrid Coating for Flame Retardant Cotton Fabrics Based on Layer-by-Layer Assembly and Sol-Gel Process. RSC $A d v$. 2015, 5, $10647-10655$. 
s33. Liu, Y.; Cao, Z. H.; Zhang, Y.; Fang, P. Synthesis of Zinc N-Morpholinomethylphosphonic Acid and Its Application in High Density Polyethylene. Fire Saf. J. 2015, 71, 1-8.

s34. Hoffendahl, C.; Duquesne, S.; Fontaine, G.; Taschner, F.; Mezger, M.; Bourbigot, S. Decomposition Mechanism of Fire Retarded Ethylene Vinyl Acetate Elastomer (EVA) Containing Aluminum Trihydroxide and Melamine.Polym. Degrad. Stab. 2015, 113, 168-179.

s35. Ahmad, R.; Ha, J. H.; Song, I. H. Fabrication of Self-Setting $\mathrm{Al}(\mathrm{OH})_{3}$ Foams for Potential Fire-Retarding Applications. Mater. Lett. 2015, 139, 252-254.

s36. Elbasuney, S.; Mostafa, S. F. Continuous Flow Formulation and Functionalization of Magnesium Di-hydroxide Nanorods as a Clean Nano-Fire Extinguisher. Powder Technol. 2015, 278, 72-83.

s37. Costes, L.; Laoutid, F.; Dumazert, L.; Lopez-cuesta, J.; Brohez, S.; Delvosalle, C.; Dubois, P. Metallic Phytates as Efficient Bio-Based Phosphorous Flame Retardant Additives for Poly (Lactic Acid). Polym. Degrad. Stab. 2015, 119, 217-227.

s38. Chen, X. E.; Liu, L.; Jiao, C. M. Influence of Iron Oxide Brown on Smoke-Suppression Properties and Combustion Behavior of Intumescent Flame-Retardant Epoxy Composites. Adv. Polym. Technol. 2015, 34, 21516. 